\title{
International Normalized Ratio Stability in Warfarin- Experienced Patients with Nonvalvular Atrial Fibrillation
}

\author{
Winnie W. Nelson ${ }^{1} \cdot$ Sunita Desai ${ }^{2} \cdot$ Chandrasekharrao V. Damaraju $^{3} \cdot$ \\ Lang Lu $^{3} \cdot$ Larry E. Fields ${ }^{4} \cdot$ Peter Wildgoose $^{4} \cdot$ Jeffery R. Schein ${ }^{1}$
}

Published online: 6 May 2015

(C) The Author(s) 2015. This article is published with open access at Springerlink.com

\begin{abstract}
Background Maintaining stable levels of anticoagulation using warfarin therapy is challenging. Few studies have examined the stability of the international normalized ratio (INR) in patients with nonvalvular atrial fibrillation (NVAF) who have had $\geq 6$ months' exposure to warfarin anticoagulation for stroke prevention.

Objective Our objective was to describe INR control in NVAF patients who had been receiving warfarin for at least 6 months.

Methods Using retrospective patient data from the CoagClinic $^{\mathrm{TM}}$ database, we analyzed data from NVAF patients treated with warfarin to assess the quality of INR control and possible predictors of poor INR control. Time within, above, and below the recommended INR range (2.0-3.0) was calculated for patients who had received warfarin for $\geq 6$ months and had three or more INR values. The analysis also assessed INR patterns and resource utilization of patients with an INR >4.0. Logistic regression models were used to determine factors associated with poor INR control.

Results Patients $(n=9433)$ had an average of 1.6 measurements per 30 days. Mean follow-up time was 544 days. Approximately $39 \%$ of INR values were out of range, with $23 \%$ of INR values being $<2.0$ and $16 \%$ being $>3.0$. Mean percent time with INR in therapeutic range was $67 \%$; INR
\end{abstract}

Winnie W. Nelson

wnelson@its.jnj.com

1 Health Economics and Outcomes Research (HECOR), Janssen Scientific Affairs, LLC, 1000 US Highway 202 South, Raritan, NJ 08869, USA

2 CTI/S2 Statistical Solutions, Cincinnati, OH, USA

3 Janssen R\&D, LLC, Raritan, NJ, USA

4 Janssen Pharmaceuticals, Inc., Raritan, NJ, USA
$<2.0$ was $19 \%$ and INR $>3.0$ was $14 \%$. Patients with more than one reading of INR $>4.0(\sim 39 \%)$ required an average of one more visit and took 3 weeks to return to an in-range INR. Male sex and age $>75$ years were predictive of better INR control, whereas a history of heart failure or diabetes were predictive of out-of-range INR values. However, patient characteristics did not predict the likelihood of INR $>4.0$.

Conclusions Out-of-range INR values remain frequent in patients with NVAF treated with warfarin. Exposure to high INR values was common, resulting in increased resource utilization.

\section{Key Points}

In patients with nonvalvular atrial fibrillation, maintaining stable, therapeutic levels of anticoagulation for stroke prevention is challenging.

Even after 6 months of warfarin therapy, approximately $40 \%$ of international normalized ratio (INR) values were out of the therapeutic range of 2.0-3.0. In addition, patients exposed to INR $>4.0$ consumed more healthcare resources.

Patient characteristics were not predictive of increased risk of over-anticoagulation, emphasizing the unpredictability of warfarin.

\section{Introduction}

Atrial fibrillation (AF) is a common and clinically significant cardiac arrhythmia. It is estimated that 2.3 million Americans have $\mathrm{AF}[1,2]$, and its prevalence is expected to 
increase 2.5-fold by 2050 [1]. AF is an important concern to the healthcare system, because it increases the risk of stroke up to fivefold [3], and AF-related stroke is typically more severe and carries a higher mortality rate than stroke not associated with $\mathrm{AF}$ [4].

Increased time in therapeutic range (TTR) correlates with improved patient outcomes and lower costs [5]. Longterm anticoagulants are recommended by guidelines for the prevention of stroke among high-risk AF patients [6], and warfarin has been widely used for stroke prevention in such patients with nonvalvular AF (NVAF). A 2007 meta-analysis of 13 randomized clinical trials showed that doseadjusted warfarin reduced the relative risk of stroke by $64 \%$ compared with placebo [7]. Achieving stable international normalized ratio (INR) values is critical but can be challenging. In a recent study that examined INR patterns in more than 15,000 patients with NVAF newly initiated on warfarin, $25 \%$ of patients failed to achieve INR stabilization during their treatment, and most of these patients ultimately discontinued warfarin therapy [8].

While several studies have explored the difficulties of achieving target INR values among patients who are new to warfarin therapy, few studies have focused on the experience of patients who have overcome the initial hurdles of INR control and have used warfarin for more than 6 months. Furthermore, few studies have examined determinants of INR control. Of those that have, most have focused only on the occurrence of sub-therapeutic INR values (i.e., INR $<2.0$ ) and have not examined the occurrence and impact of supra-therapeutic INR values (i.e., INR $>3.0$ ).

Therefore, the objective of the current study was to describe INR control in NVAF patients who had been receiving warfarin for at least 6 months. We examined the occurrence of both sub-therapeutic and supra-therapeutic INR values in this population and the management patterns in response to readings of INR $\geq 4.0$. In addition, we assessed predictors of poor INR control among 'experienced' warfarin patients.

\section{Methods}

\subsection{Data and Study Design}

This was a retrospective analysis of a database of patientlevel anticoagulation management records collected between 2006 and 2010 by CoagClinic ${ }^{\mathrm{TM}}$, a decision-support software system. CoagClinic ${ }^{\mathrm{TM}}$ software is used by a large number of institutions, mostly hospital based, in 49 states in the USA. As of December 2010, the database contained records of approximately 400,000 patients and represented the largest database of patients receiving anticoagulation therapy. The database contains demographic information, medical indication for warfarin, INR goal, INR range, medical history, detailed encounter data from each patient visit (such as date of service), and INR values. The medical history data were recorded in text and were recoded into analyzable data by the research team. The $\mathrm{CHADS}_{2}$ (congestive heart failure, hypertension, age $\geq 75$ years, diabetes mellitus, stroke/transient ischemic attack) and $\mathrm{CHA}_{2} \mathrm{DS}_{2^{-}}$ VASc (congestive heart failure, hypertension, age $\geq 75$ years, diabetes mellitus, stroke/transient ischemic attack, vascular disease, age 65-74 years, sex category) scores were calculated based on the recoded medical history data. Because the database was intended for clinical care documentation, medical history was recorded as free text. The text data were recoded into International Classification of Diseases 9th Revision, Clinical Modification codes by the research team in order to enable analytics programming.

\subsection{Eligibility Criteria}

Adults with a diagnosis of NVAF with at least three INR values and available medical history data were identified. Adults were defined as patients aged 18 years or older as of their warfarin initiation date (1 January 2006 to 31 December 2010). Patients were tracked from their first INR measurement until the end of data availability (31 December 2010) or until the occurrence of a 90-day visit gap. Patients were included in the analysis if they had at least 6 months of observable follow-up time. The warfarin initiation date was confirmed for each patient by ensuring that the year of warfarin initiation matched that of the patient's first visit date. Patients included had been using warfarin for at least 6 months and were thus considered warfarinexperienced based on the Veterans Affairs Study to Improve Anticoagulation [9]. The current analysis analyzed all INR data between the end of the first 6 months and the end of the observation period.

\subsection{Measurement of International Normalized Ratio (INR) Control}

The TTR was calculated for INR as within the recommended therapeutic range of 2.0-3.0 using the Rosendaal method [10], in which linear interpolation is used to assign an INR value to each day between successive observed INR values. This approach assumes that INR is linearly increasing or decreasing between measurements. An equivalent method that assumes linearity between INR measurements was used to calculate percent of time above and percent of time below the therapeutic INR range. In addition, percent of INR values in range or out of range was reported. This measure, which directly 
summarizes the INR values without interpolation, was deemed appropriate as a simple metric for day-to-day clinical practice [11].

\subsection{Statistical Methods: Descriptive Statistics}

Demographic characteristics reported included age and sex, and health characteristics included history of congestive heart failure, diabetes, hypertension, stroke, and vascular disease, as well as the $\mathrm{CHADS}_{2}$ and $\mathrm{CHA}_{2} \mathrm{DS}_{2}$-VASc risk stratification schemes $[12,13]$. These patient characteristics were baseline variables and were collected at the time of warfarin initiation.

Descriptive statistics on INR patterns and follow-up time were examined. Mean follow-up days, frequency of INR measurements in the sample, and percent of INR measures within, above, and below the therapeutic range were calculated. The TTR and percent of time below and above the therapeutic range were also calculated, as was the percent of patients with INR values ever out of range. Patient characteristics were stratified by quartile of percent of INR values that were out of range, with the first quartile representative of the best INR control and the fourth quartile representative of the worst INR control.

Descriptive statistics for INR patterns in 30 days following a first reading of INR $>4.0$ were examined. This included calculating the number of INR measurements in 30 days following a reading of INR $>4.0$, the amount of time to a first in-range INR reading following a reading of INR $>4.0$, and the percent of INR values below, above, and within the therapeutic range.

A logistic regression was used to determine the correlation between a high percentage of INR values out of range and various patient demographic and health characteristics. The dependent variable was binary: ' 1 ' indicated patients in the quartile with the highest percent of INR values out of range (worst INR control), and ' 0 ' indicated patients in the quartile with the lowest percent of INR values out of range (best INR control). Statistics were adjusted for age, sex, geographic region, history of congestive heart failure, diabetes, hypertension, stroke, and vascular disease.

A second logistic regression model was used to determine the correlation between any INR value $>4.0$ and patient characteristics. The dependent variable was ' 1 ' for patients who had at least one reading of INR $>4.0$ and ' 0 ' for patients who had no readings of INR $>4$.0. Statistics were adjusted for the same demographic and health characteristics as in the first regression.

The study report was written in compliance with the Strengthening the Reporting of Observational Studies in Epidemiology (STROBE) statement [14].

\section{Results}

\subsection{Descriptive Statistics}

The study sample represented 9433 patients who met the inclusion criteria and had been using warfarin for $>6$ months (Table 1). The mean age was $72.6 \pm 10.4$ years, and $46 \%$ of the sample was female. Of the patients, $12 \%$ had a history of congestive heart failure, $18 \%$ had diabetes, $51 \%$ had hypertension, $4 \%$ had stroke, and $12 \%$ had vascular disease.

\subsection{INR Patterns}

INR data starting at 6 months following warfarin initiation were examined. Mean follow-up time was $544 \pm 397$ days, and patients had an average of $1.6 \pm 0.7$ measurements per 30 days (Table 2). More than $90 \%$ of patients had at least one INR value below the therapeutic range $(<2.0)$, and $82 \%$ of patients were exposed to at least one INR $>3.0$. Moreover, $34 \%$ of patients had at least one reading between 4.0 and $5.0 ; 12 \%$ had at least one reading between 5.0 and 6.0 ; and $8 \%$ had at least one reading of INR $\geq 6.0$ (Fig. 1).

Table 1 Demographic and health characteristics

\begin{tabular}{ll}
\hline Characteristic $(N=9433)$ & Value \\
\hline Age, years & $72.6 \pm 10.4$ \\
Age group, years & $1963(20.8)$ \\
$\quad 18-65$ & $2911(30.9)$ \\
$65-75$ & $4559(48.3)$ \\
$\geq 75$ & \\
Sex & $4378(46.4)$ \\
Female & \\
Health history & $1151(12.2)$ \\
Congestive heart failure & $1731(18.4)$ \\
Diabetes & $4795(50.8)$ \\
Hypertension & $373(4.0)$ \\
Prior stroke & $1116(11.8)$ \\
$\quad$ Vascular disease & $1.4 \pm 1.0(1.0)$ \\
Mean CHADS & \pm SD (median) \\
Mean CHA ${ }_{2} \mathrm{DS}_{2}$-VASc \pm SD (median) & $2.8 \pm 3.0(1.4)$ \\
\hline
\end{tabular}

Data are presented as $n(\%)$ or mean \pm SD unless otherwise indicated $\mathrm{CHADS}_{2}$ score: congestive heart failure, hypertension, age $\geq 75$ years, diabetes mellitus, stroke/transient ischemic attack. $\mathrm{CHA}_{2} \mathrm{DS}_{2^{-}}$ VASc score: congestive heart failure, hypertension, age $\geq 75$ years, diabetes mellitus, stroke/transient ischemic attack, vascular disease, age 65-74 years, sex category

$S D$ standard deviation 
Table 2 International normalized ratio patterns

\begin{tabular}{ll}
\hline Characteristic & Value \\
\hline Mean follow-up time, days \pm SD (median) & $\begin{array}{c}544.2 \pm 396.6 \\
(455)\end{array}$ \\
Mean number of INR measurements per & $1.6 \pm 0.7(1.4)$ \\
30 days \pm SD (median) & \\
Mean \% INR values & \\
$\quad<2.0$ & 22.9 \\
$2.0-3.0$ & 61.0 \\
$>3.0$ & 16.1 \\
TTR $(\%)$ & 66.8 \\
Mean $\%$ time out of range & \\
$>2.0$ & 19.3 \\
$<3.0$ & 13.9
\end{tabular}

INR international normalized ratio, $S D$ standard deviation, TTR time in therapeutic range

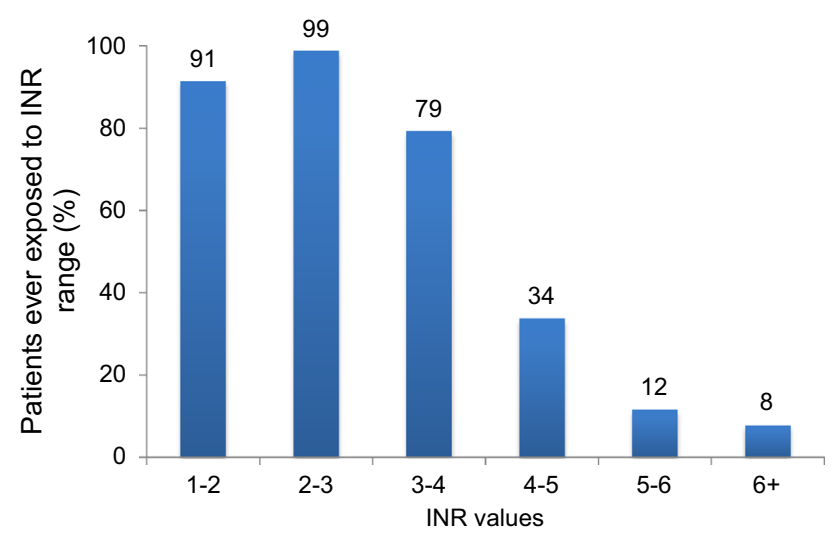

Fig. 1 Percent of patients ever exposed to INR ranges. INR international normalized ratio

\subsection{Descriptive Statistics by Quartile of Percent of Out-of-Range INR}

The quartile with the worst INR control had $64 \%$ of INR values out of range, whereas the quartile with the best INR control had $16 \%$ of INR values out of range (Table 3 ). Patients in the quartile with the worst INR control were, on average, 1 year younger than patients in the quartile with the best INR control. The two quartiles with better INR control contained more male patients. Patients in the quartile with the worst INR control was also more likely to have a history of congestive heart failure, diabetes, and/or vascular disease; however, this group did not differ from the other groups with regard to $\mathrm{CHADS}_{2}$ or $\mathrm{CHA}_{2} \mathrm{DS}_{2}$ VASc scores.

\subsection{Descriptive Statistics for INR Patterns in 30 Days Following First INR $>4.0$}

Approximately $39 \%$ of the sample $(n=3687)$ had at least one INR value $>4.0$. In the 30 days following a reading of INR $>4.0$, patients had on average 2.5 INR measurements (range 1-12), during which time approximately $24 \%$ of INR values were $<2.0$, and $23 \%$ of values were $>3.0$ (Table 4). The next in-range INR value was checked after an average of 21 days following the reading of INR $>4.0$.

\subsection{Logistic Regression of Patient Characteristics in Patients with the Worst INR Control Versus Patients with the Best INR Control by Quartile of Percent INR Values Out of Range (Patients in the Best and Worst Quartiles Only)}

Figure 2 shows the adjusted odds ratios (ORs) and $95 \%$ confidence intervals (CIs) for patient characteristics as they are associated with better or worse INR control. Male sex (OR 0.70, $95 \%$ CI 0.62-0.79), older age (OR 0.85, $95 \%$ CI 0.75-0.96), and hypertension (OR 0.73, $95 \%$ CI 0.64-0.83) were associated with better INR control. Heart failure (OR $1.72,95 \%$ CI 1.43-2.08) and diabetes (OR 1.21, $95 \%$ CI $1.03-1.42)$ were associated with worse INR control.

\subsection{Logistic Regression of Characteristics of Patients Ever Having INR Values >4.0}

The only statistically significant coefficients were geography and sex (Fig. 3). Male sex (OR 0.83, $95 \%$ CI 0.74-0.94) and living in the northeast versus the west (OR 0.77 , $95 \%$ CI $0.63-0.94$ ) were associated with lower likelihood of exposure to INR >4.0. Living in the south versus the west (OR 1.35, $95 \%$ CI 1.10-1.65) was associated with a higher likelihood of exposure to INR $>4.0$. Coefficients for the other demographic and health characteristics were not statistically significant.

\section{Discussion}

Studies of newer oral anticoagulants have demonstrated that warfarin-experienced patients have unique characteristics and experience different outcomes than do newly initiated patients [15]. In addition, a recent publication by Schneeweiss et al. [16] cautioned on the risk of confounding bias when conducting real-world analyses combining data from new and experienced users of anticoagulants. Because the majority of real-world studies of INR stability in patients with AF focus on newly 
Table 3 Descriptive statistics by quartile of international normalized ratio values out of range ${ }^{\mathrm{a}}$

\begin{tabular}{|c|c|c|c|c|}
\hline Characteristic & Quartile $1(n=2370)$ & Quartile $2(n=2352)$ & Quartile $3(n=2510)$ & Quartile $4(n=2201)$ \\
\hline Mean $\%$ of INR values out of range & 16.07 & 32.46 & 44.61 & 64.02 \\
\hline Age, years & $72.8 \pm 10.30$ & $73.2 \pm 9.90$ & $72.5 \pm 10.30$ & $71.7 \pm 11.10$ \\
\hline \multicolumn{5}{|l|}{ Sex } \\
\hline Female & $1000(42.2)$ & $1051(44.7)$ & $1232(49.1)$ & 1095 (49.8) \\
\hline Male & $1367(57.7)$ & $1300(55.3)$ & $1276(50.8)$ & $1104(50.2)$ \\
\hline \multicolumn{5}{|l|}{ Health history } \\
\hline Congestive heart failure & $224(9.5)$ & $259(11.0)$ & $336(13.4)$ & $332(15.1)$ \\
\hline Diabetes & $395(16.7)$ & $433(18.4)$ & $470(18.7)$ & $433(19.7)$ \\
\hline Hypertension & $1249(52.7)$ & $1249(53.1)$ & $1265(50.4)$ & 1032 (46.9) \\
\hline Prior stroke & $88(3.7)$ & $104(4.4)$ & $97(3.9)$ & $84(3.8)$ \\
\hline Vascular disease & $253(10.7)$ & $258(11.0)$ & $298(11.9)$ & $307(13.9)$ \\
\hline Mean $\mathrm{CHADS}_{2} \pm \mathrm{SD}$ (median) & $1.4 \pm 1.0(1.00)$ & $1.4 \pm 1.0(1.02)$ & $1.4 \pm 1.0(1.04)$ & $1.4 \pm 1.0(1.04)$ \\
\hline Mean $\mathrm{CHA}_{2} \mathrm{DS}_{2}$-VASc $\pm \mathrm{SD}$ (median) & $2.7 \pm 3.0(1.40)$ & $2.8 \pm 3.0(1.39)$ & $2.8 \pm 3.0(1.46)$ & $2.7 \pm 3.0(1.45)$ \\
\hline
\end{tabular}

Data are presented as mean $\pm \mathrm{SD}$ or $n(\%)$ unless otherwise indicated

Sex data were not available for eight individuals

$I N R$ international normalized ratio, $S D$, standard deviation

${ }^{\text {a }}$ Quartile 1 = best INR control; Quartile 4 = worst INR control

Table 4 INR patterns in the 30 days following an international normalized ratio $>4.0$

\begin{tabular}{lc}
\hline INR pattern assessed & Value \\
\hline Number of INR values in 30 days after INR $>4.0$ & $2.5 \pm 1.4(2.0)$ \\
Days to next in-range after INR $>4.0$ & $21.2 \pm 23.5(14.0)$ \\
$\%$ INR $<2$ in 30 days after INR $>4.0$ & $24.2 \pm 31.8(0.0)$ \\
$\%$ INR $>3$ in 30 days after an INR $>4.0$ & $23.4 \pm 31.0(0.0)$ \\
\hline
\end{tabular}

Data are presented as mean \pm SD (median)

$I N R$ international normalized ratio, $S D$ standard deviation

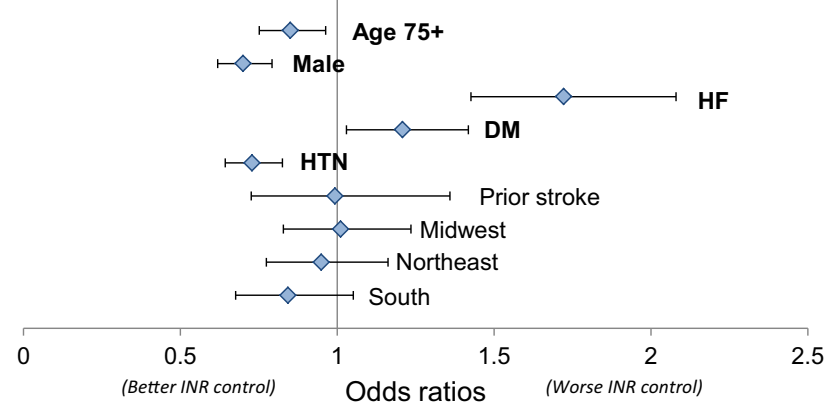

Fig. 2 Logistic regression of best versus worst international normalized ratio (INR) control by quartile of patients with out-of-range INR values on demographic and health characteristics. An odds ratio $>1$ indicated an association with worse INR control, and an odds ratio of $<1$ indicated an association with better INR control. Reference region with which midwest, northeast, and south were compared: west region. $D M$ diabetes mellitus, $H F$ heart failure, $H T N$ hypertension, $I N R$ international normalized ratio

initiated patients, a gap in the knowledge base exists with regard to the extent of INR control in patients experienced with anticoagulation therapy.

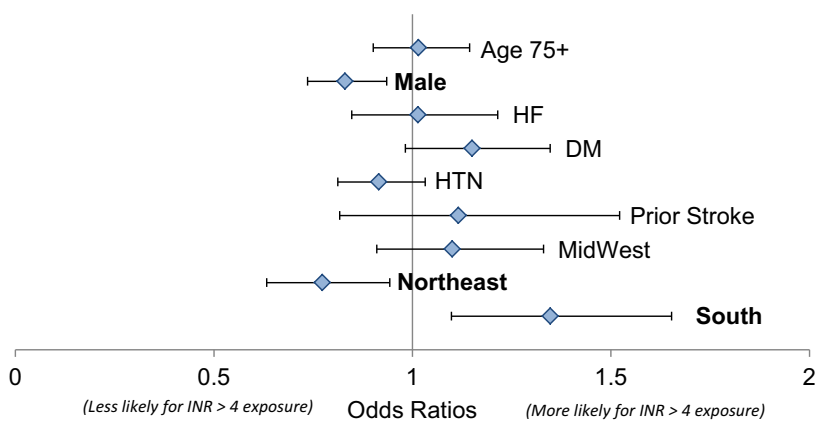

Fig. 3 Logistic regression of characteristics of patients ever exposed to international normalized ratio (INR) $>4.0$. An odds ratio $>1$ indicates a higher likelihood of exposure to INR $>4.0$. DM diabetes mellitus, $H F$ heart failure, $H T N$ hypertension. Reference region to which midwest, northeast, and south were compared: west region

This study examined INR control in warfarin-experienced patients with NVAF-those who had been using warfarin for at least 6 months at study entry. For the average 
patient, four of ten INR values were out of the therapeutic range of 2.0-3.0. Exposure to out-of-range INR was substantial: $91 \%$ of the cohort had at least one INR $<2.0$ and $82 \%$ had an INR $>3.0$. We observed a large variation in the experience of warfarin users, with the best and the worst quartiles of patients having 16 versus $64 \%$ of out-of-range INR values, respectively. Approximately $39 \%$ of our sample had exposure to INR $>4.0$ at least once.

Patients exposed to high INR values consumed greater healthcare resources: in the 30 days following the reading of INR $>4.0$, patients had an average of 2.5 INR measurements, compared with 1.6 INR measurements for the full sample. Certain demographic and health characteristics, such as female sex, age $<75$ years, and a history of heart failure or diabetes, predicted having a high percentage of out-of-range INR values.

Our analyses showed that even among patients who have overcome the initial hurdles associated with warfarin use, maintaining in-range INR values remains challenging, with $39 \%$ of INR values being out of range, and INR values being out of range $33 \%$ of the time. Over $90 \%$ of patients experienced out-of-range INR values, indicating that very few patients were stable and in-range over time. In addition, a large portion of patients were exposed to INR $>4.0$; such high INR values indicate exposure to higher bleeding risk. We also observed that these patients consumed more healthcare resources, with an average of one additional follow-up visit in the following month, compared with the overall cohort. For these patients, it took approximately 3 weeks on average to establish an in-range INR reading. While our analysis revealed some predictors for poor INR control in general, we found that demographic and health characteristics were not helpful in predicting likelihood of exposure to supra-therapeutic INR values. Most likely, this is because a high INR reading may be induced by acute events such as worsening of comorbidities or changes in diet or other medication use.

Our results corroborate previous findings in the literature related to the difficulty of achieving INR control. Rose et al. [9] studied determinants of INR control separately among patients in the first 6 months and after 6 months of warfarin use. This analysis of experienced warfarin users included about 100,000 patients in the Veterans Health Administration system. The average TTR among this group was $61 \%$, which is very similar to the TTR of $67 \%$ in the current study. This study used TTR as its dependent variable of interest and examined how a number of risk factors predict TTR. According to Rose et al. [9], predictors of low TTR among these patients included female sex, a greater number of hospitalizations, use of more medications, alcohol abuse, cancer, dementia, non-alcohol substance abuse, and chronic liver disease. Our study focused on fewer risk factors while adding to the literature by examining patterns of INR in more detail, including a description of the occurrence of sub-therapeutic and supra-therapeutic INR values and the impact of very high INR values on anticoagulation management. Our findings were also similar to those of a metaanalysis conducted by Baker and colleagues [5] examining INR control in patients with AF managed in an anticoagulation clinic versus in a community setting. The mean TTR in patients managed in an anticoagulation clinic was $63 \%$ compared with $51 \%$ for patients managed in a community practice.

A study conducted in the Netherlands focused on health characteristics and INR patterns once patients had reached stability [17]. This study included patients receiving vitamin $\mathrm{K}$ antagonists (e.g., warfarin) at an anticoagulation clinic regardless of indication. INR stability was defined as four consecutive in-range INR values (in-range was defined as 2.0-3.5 for low-intensity treatment and 2.5-4.0 for highintensity treatment). Average time to stabilization was 12 weeks. At 4 weeks after stabilizing, $12 \%$ of patients had a risk of sub-therapeutic INR values. This percentage of patients increased to $21 \%$ after 8 weeks and $50 \%$ after 40 weeks. While these investigators found that $50 \%$ of patients were at risk of exposure to sub-therapeutic INR values 40 weeks following stabilization [17], we found a much higher percentage: after 6 months of warfarin use, $>90 \%$ of patients had a sub-therapeutic INR during an average of 78 weeks of observation. This difference may be related to the definitions of in-range INR values: specifically, while we use a fixed therapeutic range, Rombouts et al. [17] used different ranges depending on whether a patient was deemed appropriate for low- or highintensity treatment.

Witt et al. [18] conducted a retrospective analysis of approximately 7500 patients to study determinants of INR stabilization, defined as an INR value within the therapeutic range continuously for a 6-month period. A total of $33 \%$ of the sample achieved stabilization. Predictors of stabilization were older age ( $>70$ years) and having no comorbid heart failure or diabetes. Consistent with Witts et al. [18], we found that older age correlated with better INR control, as did not having comorbid congestive heart failure.

\subsection{Limitations}

This analyses has several limitations. The data were generalizable only to patients managed in anticoagulation clinics. INR patterns might differ in patients who are managed elsewhere. Observational data might contain inaccuracies because of errors in recording the information. The extended follow-up period might have minimized the impact of an occasional inaccurate INR reading. The analytic models used in our study included variables available in the data but 
could not adjust for unobserved patient characteristics such as diet, concomitant medication, and certain comorbidities. Therefore, residual confounding may exist. The available data allowed patients to be followed until they discontinued anticoagulation management clinic visits. The reasons for discontinuation were not part of the dataset, nor were clinical outcomes. Therefore, the current analysis was limited to describing INR patterns and their associations with certain patient characteristics, with no assumptions regarding patient disposition.

\section{Conclusions}

Our analysis examined INR control among patients who had at least 6 months of warfarin experience and found evidence of poor INR control even after this longer-term use. A significant portion of the patient cohort experienced an INR $\geq 4.0$, with an associated increase in follow-up visits. Moreover, we found that, while certain patient characteristics were predictors of poor INR control, these characteristics could not predict who was at increased risk of high INR values, further emphasizing the unpredictability of warfarin therapy. More research is needed to determine the clinical and economic outcomes of thrombosis and bleeding events associated with exposure to outof-range INR values.

Acknowledgments The authors would like to acknowledge Lisa Grauer, MSc, who provided editorial support with funding from Janssen Scientific Affairs, LLC.

Financial disclosure This study was funded by Janssen Scientific Affairs, LLC, Raritan, NJ, USA.

Conflict of interest WWN and JRS are employees of Janssen Scientific Affairs, LLC (a J\&J company); SD is a consultant of CTI/ S2 Statistical Solutions, a paid consultant to Janssen Scientific Affairs; CVD and LL are employees of Janssen R\&D, LLC (a J\&J company); and LEF and PW are employees of Janssen Pharmaceuticals, Inc. (a J\&J company). All J\&J authors are shareholders of J\&J.

Open Access This article is distributed under the terms of the Creative Commons Attribution-NonCommercial 4.0 International License (http://creativecommons.org/licenses/by-nc/4.0/), which permits any noncommercial use, distribution, and reproduction in any medium, provided you give appropriate credit to the original author(s) and the source, provide a link to the Creative Commons license, and indicate if changes were made.

\section{References}

1. Go AS, Hylek EM, Phillips KA, et al. Prevalence of diagnosed atrial fibrillation in adults. National implications for rhythm management and stroke prevention: the AnTicoagulation and Risk Factors in Atrial Fibrillation (ATRIA) study. JAMA. 2001;285:2370-5.
2. Feinberg WM, Blackshear JL, Laupacis A, Kronmal R, Hart RG. Prevalence, age distribution, and gender of patients with atrial fibrillation. Analysis and implications. Arch Intern Med. 1995;155:469-73.

3. Wolf PA, Abbott RD, Kannell WB. Atrial fibrillation: a major contributor to stroke in the elderly: the Framingham Study. Arch Intern Med. 1987;147:1561-4.

4. Jorgensen HS, Nakayama H, Reith J, Raaschou HO, Olsen TS. Acute stroke with atrial fibrillation: The Copenhagen Stroke Study. Stroke. 1996;10:1765-9.

5. Baker WL, Cios DA, Sander SD, Coleman CI. Meta-analysis to assess the quality of warfarin control in atrial fibrillation patients in the United States. J Manag Care Pharm. 2009;15:244-52.

6. January CT, Wann LS, Alpert JS, Calkins H, Cigarroa JE, Cleveland JC Jr, Conti JB, Ellinor PT, Ezekowitz MD, Field ME, Murray KT, Sacco RL, Stevenson WG, Tchou PJ, Tracy CM, Yancy CW. American College of Cardiology/American Heart Association Task Force on Practice Guidelines. 2014 AHA/ACC/ HRS guideline for the management of patients with atrial fibrillation: a report of the American College of Cardiology/American Heart Association Task Force on Practice Guidelines and the Heart Rhythm Society. J Am Coll Cardiol. 2014;64(21):e1-76.

7. Hart RG, Pearce LA, Aguilar MI. Meta-analysis: antithrombotic therapy to prevent stroke in patients who have nonvalvular atrial fibrillation. Ann Intern Med. 2007;146:857-67.

8. Nelson WW, Desai S, Damaraju CV, Lu L, Fields LE, Wildgoose P, Schein JR. International normalized ratio stabilization in newly initiated warfarin patients. Curr Med Res Opin. 2014;30(12):2437-42.

9. Rose AJ, Hylek EM, Ozonoff A, Ash AS, Reisman JI, Berlowitz DR. Patient characteristics associated with oral anticoagulation control: results of the Veterans AffaiRs Study to Improve Anticoagulation (VARIA). J Thromb Haemost. 2010;8:2181-91.

10. Rosendaal FR, Cannegieter SC, van der Meer FJ, Briet E. A method to determine the optimal intensity of oral anticoagulant therapy. Thromb Haemost. 1993;69:236-9.

11. Schmitt L, Speckman J, Ansell J. Quality assessment of anticoagulation dose management: comparative evaluation of measures of time-in-therapeutic range. J Thromb Thrombolys. 2003;15:213-6.

12. Gage BF, Waterman AD, Shannon W, Boechler M, Rich MW, Radford MJ. Validation of clinical classification schemes for predicting stroke: results from the National Registry of Atrial Fibrillation. JAMA. 2001;285:2864-70.

13. Lip GY, Nieuwlaat R, Pisters R, Lane DA, Crijns HJ. Refining clinical risk stratification for predicting stroke and thromboembolism in atrial fibrillation using a novel risk factor-based approach: the euro heart survey on atrial fibrillation. Chest. 2010;137:263-72.

14. von Elm E, Altman DG, Egger M, Pocock SJ, Gøtzsche PC, Vandenbroucke JP, STROBE Initiative. Strengthening the Reporting of Observational Studies in Epidemiology (STROBE) statement: guidelines for reporting observational studies. BMJ. 2007;335:806-8.

15. Mahaffey KW, Wojdyla D, Hankey GJ, et al. Clinical outcomes with rivaroxaban in patients transitioned from vitamin $\mathrm{K}$ antagonist therapy: a subgroup analysis of a randomized trial. Ann Intern Med. 2013;158:861-8.

16. Schneeweiss S, Huybrechts KF, Gagne JJ. Interpreting the quality of health care database studies on the comparative effectiveness of oral anticoagulants in routine care. Comp Eff Res. 2013;3:33-31.

17. Rombouts EK, Rosendaal FR, van der Meer FJ. Subtherapeutic oral anticoagulant therapy: frequency and risk factors. Thromb Haemost. 2009;101:552-6.

18. Witt DM, Delate T, Clark NP, et al. Warfarin Associated Research Projects and other EnDeavors (WARPED) Consortium. Outcomes and predictors of very stable INR control during chronic anticoagulation therapy. Blood. 2009;114:952-6. 